\title{
Gastric syphilis - Case report ${ }^{\star}$
}

\section{Tais Ferreira Guimarães ${ }^{1}$ \\ Caroline Bertolini Bottino ${ }^{1}$ Ricardo Barbosa Lima ${ }^{1}$}

\author{
Camila Freitas Lobo Novis ${ }^{1}$ \\ Antonio Macedo D' Acri ${ }^{1}$ \\ Carlos José Martins ${ }^{1}$
}

DOI: http:/ / dx.doi.org/10.1590/abd1806-4841.20164174

\begin{abstract}
Gastric syphilis is an uncommon extracutaneous manifestation of syphilis, occurring in less than $1 \%$ of patients, presenting nonspecific clinical manifestations. In general, it occurs on secondary stage. The critical point is the recognition of the syphilitic gastric involvement, without which there may be incorrect diagnosis of malignancy of the digestive tract. In this report, a case of secondary syphilis with gastric involvement that had complete remission with benzathine penicillin will be described.
\end{abstract}

Keywords: Gastritis; Syphilis; Treponema pallidum

\section{INTRODUCTION}

Syphilis is a contagious infectious disease caused by Treponema pallidum, transmitted mainly sexually, in which the agent penetrates the skin after small abrasions. Soon after, there is hematogenic dissemination, at which point any organ may be affected by the disease - among them, the stomach.

Gastric syphilis (GS), although not frequently remembered outside the scope of gastroenterology, has been a theme of medical studies since 1834, when Andral published the first two cases of patients with syphilis and gastric symptoms, which improved through treatment available at the time. ${ }^{1}$

Gastric involvement occurs in less than 1\% of syphilis cases, with the antrum as the most affected site. Average age of the affected is 39 , with predominance of males. Around $60 \%$ of patients with GS have current or previous history of syphilis. ${ }^{2}$ In the present case, we will describe a case of a patient with secondary syphilis and gastric involvement, who was diagnosed based on an investigation started by the gastroenterologist.

\section{CASE REPORT}

50-year-old male, single, looked for a gastroenterologist, complaining of epigastralgia, reported losing $6 \mathrm{~kg}$, anorexia and early satiety for the last month. He denied other gastrointestinal symptoms. Due to a suspected cancer in the digestive tube, considering his age bracket and symptoms, an upper gastrointestinal endoscopy (UGI) was requested with biopsies, which revealed antrum with edematous mucosa, infiltrated, friable, with bleeding spots and flat erosions, besides edematous mucosal pleating with foci of enanthema (Figures 1 and 2). The histopathological examination of gastric mucosa revealed a diffuse inflammatory infiltrate, mainly lymphoplasmacytic, and absence of malignancy (Figure 3).

Treponemal (FTA-Abs) and non-treponemal (VDRL = 1/32) tests were positive. The patient was referred to the Dermatology Service, and, upon examination, showed diffuse exanthema and generalized polyadenomegaly (Figures 4 and 5). He denied having noticed the hard chancre.

Considering the findings described, secondary syphilis with gastric involvement was diagnosed and the preconized treatment for it was started with benzathine penicillin (two doses of 2,400,000 IU, every seven days), as well as compulsory notification. One month later, the patient was asymptomatic and a new UGI with biopsies showed complete remission of gastric mucosa lesions (Figure 6).

\section{DISCUSSION}

GS is caused by vasculitis in the gastric mucosa generated by hematous dissemination of T. pallidum and is not linked to the ingestion of the agent. ${ }^{3}$ It may occur in any phase of syphilis, predominantly in the secondary (50\%) and more rarely in the third (6\%). ${ }^{2}$

Received on 02.11.2014.

Approved by the Advisory Board and accepted for publication on 25.01.2015.

* Work performed at the Dermatology Department of the Hospital Universitário Gaffrée e Guinle, of Universidade Federal do Estado do Rio de Janeiro (HUGGUniRio) - Rio de Janeiro (RJ), Brazil.

Financial Support: None.

Conflict of Interest: None.

1 Universidade Federal do Estado do Rio de Janeiro (UniRio) - Rio de Janeiro (RJ), Brazil.

C2016 by Anais Brasileiros de Dermatologia 


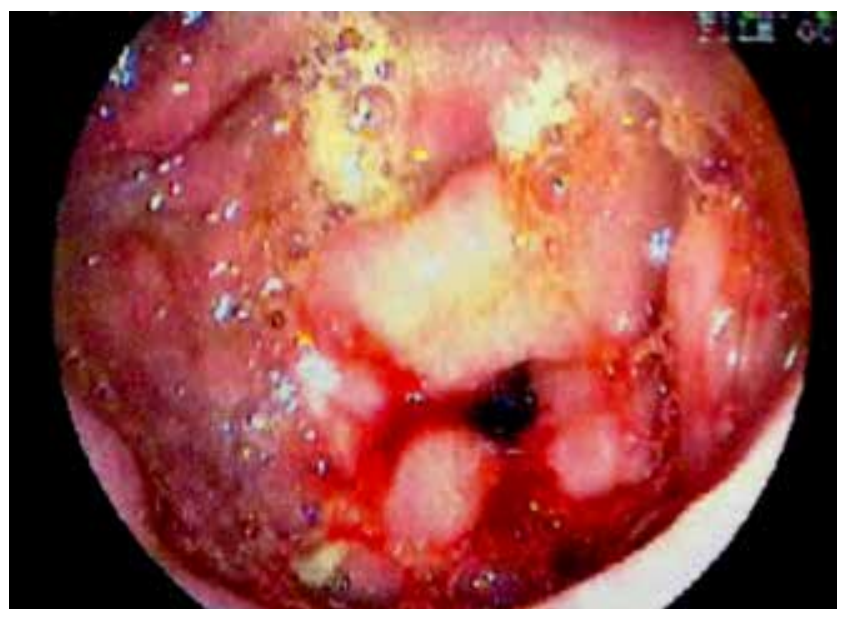

FIGURE 1: Image from upper digestive endoscopy showing antrum with edematous mucosa, infiltrated, friable, with bleeding spots and flat erosions

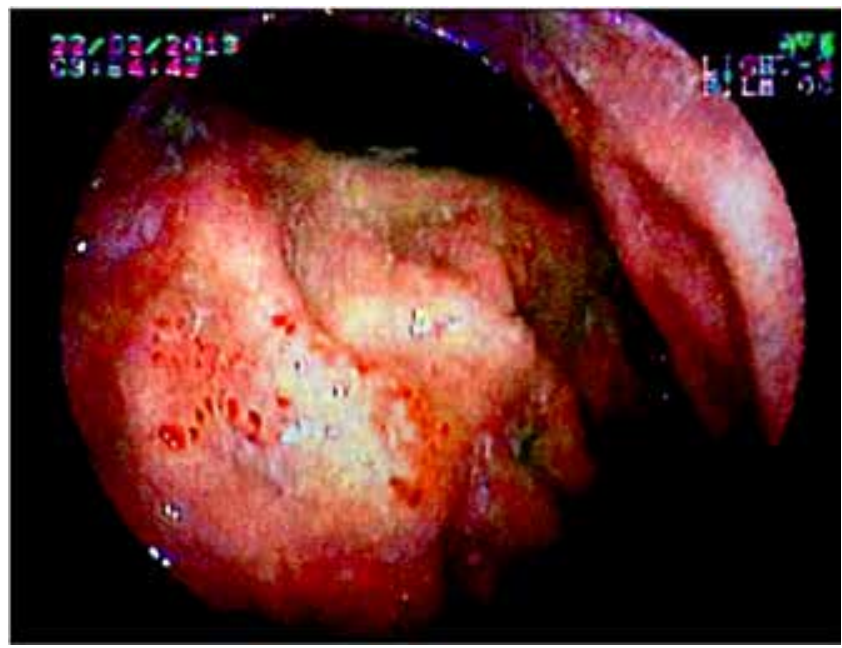

FIGURE 2: Image from upper digestive endoscopy showing edematous mucosal pleating, with foci of enanthema

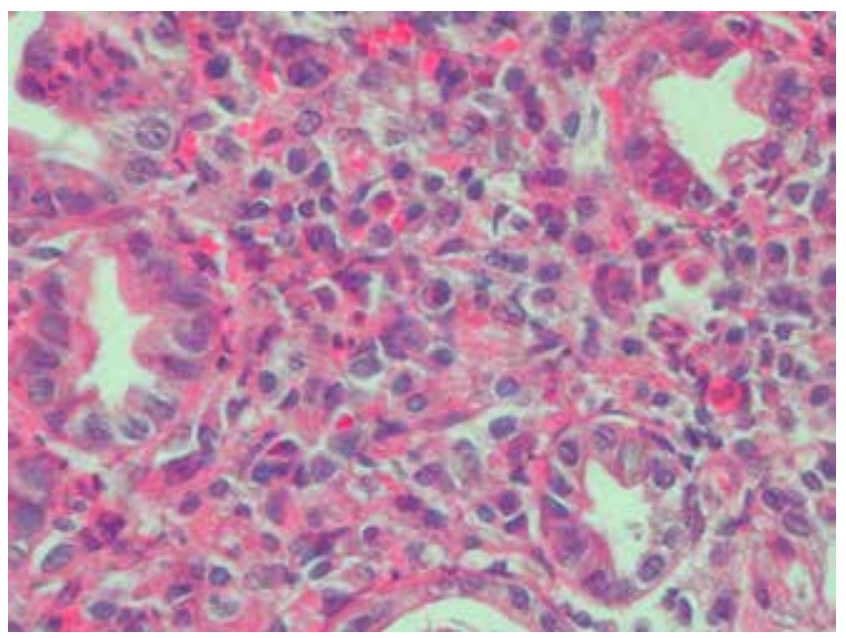

FIGURE 3: Histopathological examination of gastric mucosa revealing diffuse inflammatory infiltrate, mainly lymphoplasmacytic (HE, $400 x)$

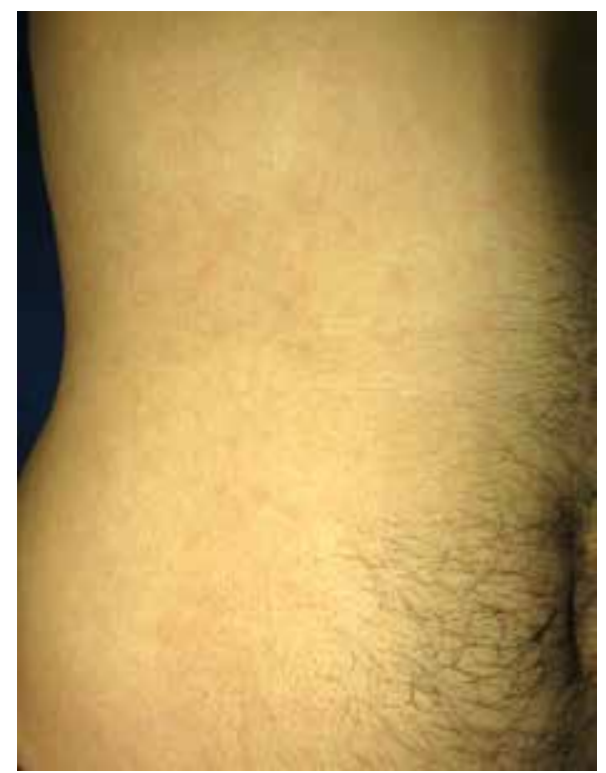

Figure 4:

Light exanthema on the flank and on the right iliac fossa

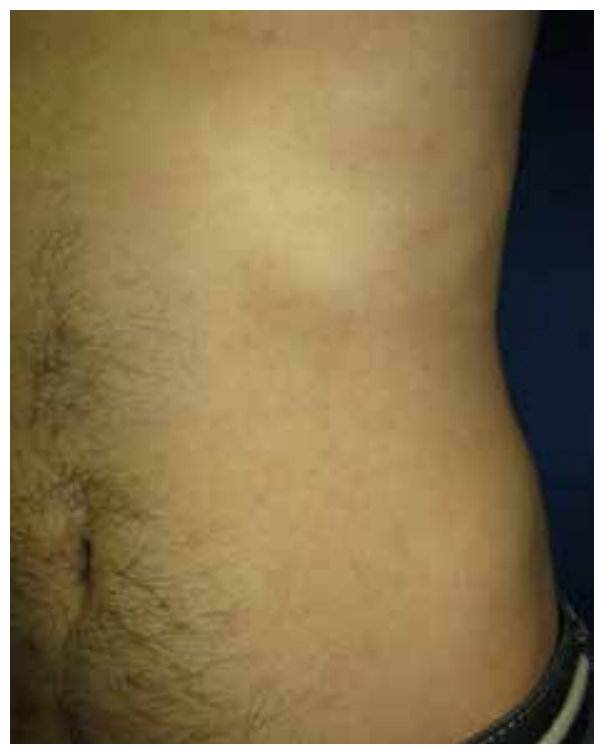

Figure 5:

Light exanthema on the left side of trunk

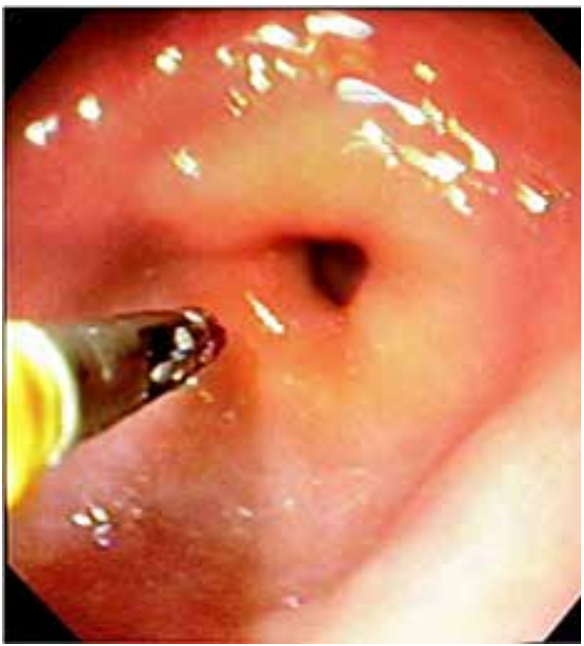

FIGURE 6:

I $m$ a $g$ e from upper d i g e s t i v e endoscopy one month after treatment with $4,800,000$ IU of benzathine penicillin 
The most common clinical manifestations of GS are epigastralgia (92\%), nausea and vomit (71\%), emaciation (60\%) and early satiety (19\%) - all unspecific symptoms. ${ }^{2}$ The absence of cutaneous lesions from syphilis does not exclude the diagnosis of GS. ${ }^{2}$

In the UGI, we observed reduction of gastric expandability, multiple ulcerations, hypertrophy of gastric walls, nodules in the mucosa, edema, enanthema and friability. GS has the following as endoscopic differential diagnoses: lymphoma, linitis plastica, tuberculosis and Crohn's disease. In these diseases, however, the lesions go beyond the pylorus, whereas in GS there is abrupt interruption of lesions in the pylorus. ${ }^{4}$

Through histopathology we observe chronic gastritis with dense lymphoplasmocytic infiltrate. ${ }^{5}$ Endovasculitis is a typical finding, although infrequent, for gastric biopsies are more superficial. There are reports in the literature of concomitant presence of Helicobacter pylori, which does not make the diagnosis invalid, but it is discussed if it acts in conjunction with the treponema, damaging the gastric mucosa. ${ }^{2}$

Detection of T. pallidum is difficult and is not mandatory for diagnosing GS. However, when it is available for PCR, it should be done, thus providing a certain diagnostic. There is the consensus that the physician should consider the patient as a carrier of GS based on the correlation of suggestive clinical findings, endoscopic image, histopathology and laboratory tests. ${ }^{6}$

For this patient, the diagnosis of syphilis was reached considering his gastric complaints, since the cutaneous manifestations were not so evident and were not the reason of his medical evaluation. Nevertheless, it was possible to observe them in the dermatological examination.

In a systematic review of GS cases reported in the last 50 years, published in 2010, Mylona et al. concluded that diagnosis of GS must be considered in patients who are in the risk group of sexually transmitted diseases and who present uncommon endoscopical lesions, without a definite diagnosis, with gastric problems resistant to pump inhibitors and with histopathological examination of gastric mucosa pointing to GS. ${ }^{2}$

The recommended treatment is the same for syphilis, according to wich phase of the disease the patient is in.

In short, this case report has the goal of showing the occurrence of GS, which is unknown by the majority of physicians. With the increasing incidence of syphilis (in the US, the rates of syphilis cases recently went up $15.2 \%$ from 2006 to 2007), mainly among men who have sexual intercourse with other men, gastric involvement may become more frequent. $\left.{ }^{7}\right]$

\section{REFERENCES}

1. Massironi S, Carmagnola S, Penagini R, Conte D. Gastric involvement in a patient with secondary syphilis. Dig Liver Dis. 2005;37:368-71.

2. Mylona EE, Baraboutis IG, Papastamopoulos V, Tsagalou EP, Vryonis E, Samarkos $M$, et al. Gastric syphilis: a systematic review of published cases of the last 50 years. Sex Transm Dis. 2010;37:177-83.

3. Kolb JC, Woodward LA. Gastric syphilis. Am J Emerg Med. 1997:15:164-6.

4. Souza Varella Frazão M, Guimarães Vilaça T, Olavo Aragão Andrade Carneiro F, Toma K, Eliane Reina-Forster C, Ryoka Baba E, et al. Endoscopic aspects of gastric syphilis. Case Rep Med. 2012;2012:646525.

5. Fyfe B, Poppiti RJ Jr, Lubin J, Robinson MJ. Gastric syphilis. Primary diagnosis by gastric biopsy: report of four cases. Arch Pathol Lab Med. 1993;117:820-3.

6. Abdu RA, Carter K, Pomidor WJ. Gastric syphilis mimicking linitis plastica. Arch Surg. 1993;128:103-4.

7. Mattei PL, Beachkofsky TM, Gilson RT, Wisco 0J. Syphilis: a reemerging infection Am Fam Physician. 2012;86:433-40
MAILING ADDRESS:

Tais Ferreira Guimarães

Rua Mariz e Barros, 775

20270-004 - Rio de Janeiro - RJ

Brazil

E-mail: taisguima@hotmail.com

How to cite this article: Guimarães TF, Novis CFL, Bottino CB, D'Acri AM, Lima RB, Martins CJ. Gastric syphilis - Case report. An Bras Dermatol. 2016;91(5):670-2. 